\title{
Emulation of an Optical Flexible Delay Line by Parallel Variable Optical Delay Lines
}

\author{
Duan-Shin Lee, Senior Member, IEEE, Cheng-Shang Chang, Fellow, IEEE, Jay Cheng, Senior Member, IEEE, \\ Hung-Shih Chueh, and Kuan-Ting Wang
}

\begin{abstract}
In this letter, we consider the discrete-time setting and propose a construction of an optical flexible delay line by parallel variable optical delay lines (VODLs) under a very simple packet routing scheme. We show that to exactly emulate an optical flexible delay line with maximum delay $d$ (time slots), where $d$ is a positive integer, it is necessary and sufficient to have at least $\lfloor(2 d+1) / 3\rfloor$ VODLs with maximum delay $d$ (time slots) in our proposed architecture. We also show that under a uniform traffic model, the average number of busy VODLs is approximately $d+1-(2 d+1)(d /(d+1))^{d}$, which is quite close to our simulation result. Through simulations, we further show that if we can tolerate a loss probability of, say $10^{-3}$, then we only need at most 1.7 times of the average number of busy VODLs, which is much smaller than the worst-case bound $\lfloor(2 d+1) / 3\rfloor$.
\end{abstract}

Index Terms-Flexible delay lines, optical buffers, optical switches, packet-switched networks, variable optical delay lines.

\section{INTRODUCTION}

$\mathbf{O}$ $\mathrm{NE}$ of the main problems in all-optical packet-switched networks is the lack of optical buffers to resolve conflicts among packets competing for the same resources in the optical domain. One feasible technology for the construction of optical buffers is to use optical crossbar Switches and fiber Delay Lines (SDL), and recently there is a surge of research results on the SDL designs of various types of optical buffers in the literature (see [1]-[2] and the references therein).

In this letter, we focus on the SDL design of optical flexible delay lines (a special type of optical buffer) [2]. As in most works in the SDL literature, we consider the discrete-time setting in which time is slotted and synchronized, and we assume that packets are of the same size so that a packet can be transmitted within a time slot (we note that variable-size bursts can be first segmented into fixed-size packets at their sources and then reassembled at their destinations). Formally, a flexible delay line with maximum delay $d$ (time slots), where $d$ is a positive integer, is a network element with one input link and one output link such that the delay of every packet is known upon its arrival and it realizes all sample paths that satisfy the following conditions:

$$
\begin{aligned}
& \tau^{a}(m) \neq \tau^{a}(n), \text { for all } m \neq n, \\
& \tau^{d}(m) \neq \tau^{d}(n), \text { for all } m \neq n, \\
& \tau^{a}(n) \leq \tau^{d}(n) \leq \tau^{a}(n)+d, \text { for all } n,
\end{aligned}
$$

The authors are with the Department of Electrical Engineering and the Institute of Communications Engineering, National Tsing Hua University, Hsinchu 30013, Taiwan, R.O.C. (e-mail: lds@cs.nthu.edu.tw, cschang@ee.nthu.edu.tw, jcheng@ee.nthu.edu.tw,d929607@oz.nthu.edu.tw, g9662521@oz.nthu.edu.tw). where $\tau^{a}(n)$ and $\tau^{d}(n)$, respectively, are the arrival time and the departure time of the $n^{\text {th }}$ packet for all $n$. We note that one application of optical flexible delay lines is to use them as the central buffers in the architecture in [2, Section VII-B] for exact emulation of an optical output-buffered switch.

In [2], it was shown that an optical flexible delay line can be constructed by a layered Cantor network. However, the routing of packets in such a layered Cantor network is very complicated. In this letter, we propose a construction of an optical flexible delay line by parallel variable optical delay lines (VODLs) under a very simple packet routing scheme. A VODL with maximum delay $d$ (time slots) is an optical delay line with adjustable delay so that its delay can be set as any delay in $\{1,2, \ldots, d\}$ and this can be done only when it is free (i.e., there are no optical packets in the VODL). When a VODL is busy (i.e., there are optical packets in the VODL), its delay cannot be adjusted. Once the delay of a VODL is set as a certain delay, say $x$, it acts like a traditional optical delay line with delay equal to $x$ so that a packet entering from its input at time $t$ will depart from its output at time $t+x$ (note that the VODL will become free at time $t+x$ if there are no other packets entering from its input during the time interval $[t+1, t+x-1])$. We note that a VODL can be implemented by using the approach based on wavelength conversion in periodically poled lithium niobate waveguides as proposed in [3].

This letter is organized as follows. In Section II, we show that to exactly emulate an optical flexible delay line with maximum delay $d$, it is necessary and sufficient to have at least $\lfloor(2 d+1) / 3\rfloor$ VODLs with maximum delay $d$ in our proposed architecture. In Section III, we show that under a uniform traffic model considered in this letter, the average number of busy VODLs is approximately $d+1-(2 d+1)(d /(d+1))^{d}$, which is quite close to our simulation result. Through simulations, we further show that if we can tolerate a loss probability of, say $10^{-3}$, then we only need at most 1.7 times of the average number of busy VODLs, which is much smaller than the worstcase bound $\lfloor(2 d+1) / 3\rfloor$. Section IV concludes this letter.

\section{EXACt EMUlation}

In this section, we show that the architecture in Figure 1 consisting of a $1 \times(M+1)$ optical crossbar switch, an $(M+1) \times 1$ optical crossbar switch, and $M$ VODLs with maximum delay $d$ can be operated as an optical flexible delay line with maximum delay $d$ under a simple packet routing scheme. Specifically, consider a sample path that satisfies the 
conditions in (1)-(3). Suppose that a packet in the sample path arrives at time $t$ and has a requested delay $x$. From the condition in (3), we have $0 \leq x \leq d$. If $x=0$, then the packet arriving at time $t$ is routed to the output link immediately via the $1 \times(M+1)$ crossbar switch, the $(M+1) \times 1$ crossbar switch, and the direct link between the two switches. On the other hand, if $1 \leq x \leq d$, then we choose a busy VODL whose delay has already been set as $x$ in the case that there are such busy VODLs, and we choose a free VODL and set its delay as $x$ in the case that there are no busy VODLs with delays set as $x$. Then the packet arriving at time $t$ is routed to the chosen VODL immediately via the $1 \times(M+1)$ crossbar switch and is routed to the output link at time $t+x$ via the $(M+1) \times 1$ crossbar switch.

As we can see from the conditions in (1) and (2) that there is at most one arrival at the input link and there is at most one departure from the output link in every time slot, it is clear that there are no conflicts among the packets at all times under our packet routing scheme. The remaining problem is whether there is always a VODL available for every arriving packet as described in our packet routing scheme, and we answer this question in the following theorem.

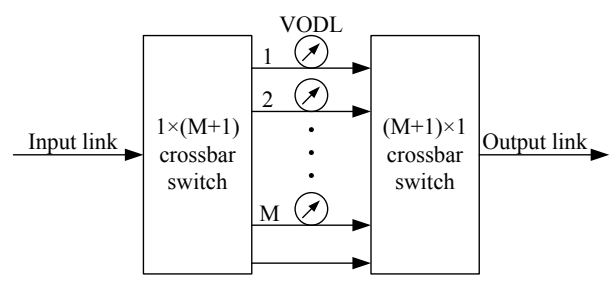

Fig. 1. Exact emulation of an optical flexible delay line with maximum delay $d$ by using $M$ parallel VODLs with maximum delay $d$.

Theorem 1 Suppose that the architecture in Figure 1 is started from an empty system. Then it can be used to exactly emulate an optical flexible delay line with maximum delay $d$ under our packet routing scheme if and only if $M \geq\left\lfloor\frac{2 d+1}{3}\right\rfloor$.

Proof. (Sufficiency) Consider a sample path that satisfies the conditions in (1)-(3). Suppose that there are $m$ busy VODLs at time $t$. As we have $M \geq\lfloor(2 d+1) / 3\rfloor$, it suffices to show that $m \leq\lfloor(2 d+1) / 3\rfloor$.

Number the $m$ busy VODLs at time $t$ from 1 to $m$ and choose a packet from each of the $m$ busy VODLs at time $t$. Let $\tau_{i}^{a}, \tau_{i}^{d}$, and $d_{i}$, respectively, be the arrival time, the departure time, and the delay of the packet chosen from the $i^{\text {th }}$ busy VODL at time $t$ for $i=1,2, \ldots, m$. Since these packets must have arrived at the VODLs by time $t$ and have not departed from the VODLs at or before time $t$, we have

$$
\tau_{i}^{a} \leq t<\tau_{i}^{d}, \text { for } i=1,2, \ldots, m .
$$

Let $d_{i}^{\prime}=t-\tau_{i}^{a}$ and $d_{i}^{\prime \prime}=\tau_{i}^{d}-t$ for $i=1,2, \ldots, m$, and let $d_{(i)}^{\prime}\left(\right.$ resp., $\left.d_{(i)}^{\prime \prime}\right)$ be the $i^{\text {th }}$ smallest element in $\left\{d_{1}^{\prime}, d_{2}^{\prime}, \ldots, d_{m}^{\prime}\right\}$ (resp., $\left\{d_{1}^{\prime \prime}, d_{2}^{\prime \prime}, \ldots, d_{m}^{\prime \prime}\right\}$ ) for $i=1,2, \ldots, m$. As it is easy to see from (1) and (4) that $d_{1}^{\prime}, d_{2}^{\prime}, \ldots, d_{m}^{\prime}$ are distinct nonnegative integers, and from (2) and (4) that $d_{1}^{\prime \prime}, d_{2}^{\prime \prime}, \ldots, d_{m}^{\prime \prime}$ are distinct positive integers, we deduce that

$$
d_{(i)}^{\prime} \geq i-1 \text { and } d_{(i)}^{\prime \prime} \geq i, \text { for } i=1,2, \ldots, m \text {. }
$$

Since it is clear that $d_{i}=\tau_{i}^{d}-\tau_{i}^{a}=\left(t-\tau_{i}^{a}\right)+\left(\tau_{i}^{d}-t\right)=d_{i}^{\prime}+d_{i}^{\prime \prime}$ for $i=1,2, \ldots, m$, it then follows from (5) that

$$
\begin{aligned}
\sum_{i=1}^{m} d_{i} & =\sum_{i=1}^{m}\left(d_{i}^{\prime}+d_{i}^{\prime \prime}\right)=\sum_{i=1}^{m} d_{(i)}^{\prime}+\sum_{i=1}^{m} d_{(i)}^{\prime \prime} \\
& \geq \sum_{i=1}^{m}(i-1)+\sum_{i=1}^{m} i=m^{2} .
\end{aligned}
$$

Let $d_{[i]}$ be the $i^{\text {th }}$ largest element in $\left\{d_{1}, d_{2}, \ldots, d_{m}\right\}$ for $i=1,2, \ldots, m$. As it is easy to see from our packet routing scheme that $1 \leq d_{i} \leq d$ for $i=1,2, \ldots, m$ and the delays of the $m$ busy VODLs at time $t$ are different, i.e., $d_{1}, d_{2}, \ldots, d_{m}$ are distinct, we deduce that

$$
d_{[i]} \leq d-i+1, \text { for } i=1,2, \ldots, m .
$$

It then follows from (7) that

$$
\sum_{i=1}^{m} d_{i}=\sum_{i=1}^{m} d_{[i]} \leq \sum_{i=1}^{m}(d-i+1)=m d-\frac{m(m-1)}{2} .
$$

From (6) and (8), we immediately obtain $m \leq(2 d+1) / 3$, which clearly implies that $m \leq\lfloor(2 d+1) / 3\rfloor$.

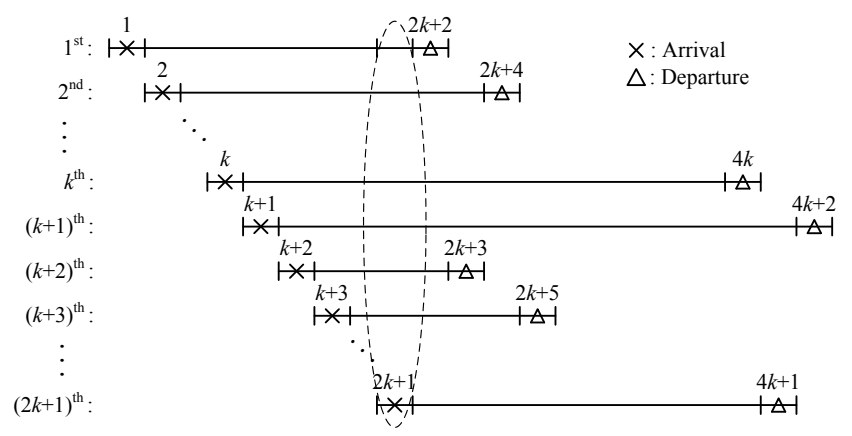

Fig. 2. The sample path in the proof of "necessity" of Theorem 1.

(Necessity) We prove the necessity by giving a sample path that satisfies the conditions in (1)-(3) and requires at least $\lfloor(2 d+1) / 3\rfloor$ VODLs, i.e., $M \geq\lfloor(2 d+1) / 3\rfloor$, under our packet routing scheme.

Suppose that $d=3 k+1$ for some $k \geq 0$ (the proof for the case that $d=3 k$ for some $k \geq 1$ or $d=3 k+2$ for some $k \geq 0$ is similar). Consider the sample path (see Figure 2 for an illustration) with

$$
\begin{aligned}
\tau^{a}(n) & =n, \text { for } n=1,2, \ldots, 2 k+1, \\
\tau^{d}(n) & =\left\{\begin{array}{l}
2 k+2 n, \text { for } n=1,2, \ldots, k+1, \\
2 n-1, \text { for } n=k+2, k+3, \ldots, 2 k+1 .
\end{array}\right.
\end{aligned}
$$

It is easy to see from (9) and (10) that the sample path satisfies the conditions in (1)-(3). As it is also easy to see that $\tau^{a}(n) \leq$ $2 k+1<\tau^{d}(n)$ for $n=1,2, \ldots, 2 k+1$ and the delays of the $2 k+1$ packets in the sample path are distinct, it follows from our packet routing scheme that the $2 k+1$ packets in the sample path are in different busy VODLs at time $t=2 k+1$. Therefore, we must have $M \geq 2 k+1=\lfloor(2 d+1) / 3\rfloor$. (Note that if we let $t=2 k+1$ and let $\tau_{i}^{a}, \tau_{i}^{d}, d_{i}, d_{i}^{\prime}, d_{i}^{\prime \prime}, d_{(i)}^{\prime}, d_{(i)}^{\prime \prime}$, and $d_{[i]}$ be defined as in the proof of "sufficiency" for $i=1,2, \ldots, 2 k+1$, then it can be seen that all of the inequalities in (5)-(8) (with $m$ replaced by $2 k+1$ ) hold with equalities.) 


\section{Number OF Busy VODLS}

In this section, we show that the number of busy VODLs could be much smaller than the worst-case bound $\lfloor(2 d+1) / 3\rfloor$ given in Theorem 1. We consider the following uniform traffic model: (i) There is exactly one packet arrival in every time slot. (ii) The delay requested by any arriving packet is uniformly distributed over $\{0,1, \ldots, d\}$ and this is independent of everything else. (iii) If an arriving packet requests to depart at a time that has already been scheduled for departure of an earlier arriving packet, then the later arriving packet is not admitted into the optical flexible delay line and is lost. It is clear from (i), (iii), and (ii) that the conditions in (1), (2), and (3), respectively, are satisfied.

We first calculate the average number of busy VODLs for the uniform traffic model. For this, we need to show that the probability that an arriving packet requesting delay $i$ is admitted into the flexible delay line is given by $p_{i}=(d /(d+1))^{d-i}$ for $1 \leq i \leq d$. To see this, consider the packet arriving at time $t$, called the tagged packet, and assume that it requests delay $i$. As there is exactly one arriving packet in every time slot (property (i)) and the delay requested by any arriving packet is at most $d$ (property (ii)), it is clear from property (iii) that the tagged packet is admitted into the flexible delay line if and only if the packet arriving at time $t-j$ does not request delay $j+i$ for all $j=1,2, \ldots, d-i$ (i.e., no packets arriving before time $t$ are scheduled to depart at time $t+i$ ). As such, it follows from the independent uniform delay distribution in property (ii) that $p_{i}=(d /(d+1))^{d-i}$.

Let $N(t)$ be the number of busy VODLs at time $t$ and let $B_{t, i}$ be the event that there is a busy VODL at time $t$ whose delay is set as $i$. As the delays of the busy VODLs at any time are different under our packet routing scheme, it follows that $N(t)=\sum_{i=1}^{d} I_{B_{t, i}}$, where $I_{B_{t, i}}$ is the indicator function of the event $B_{t, i}$, i.e., $I_{B_{t, i}}=1$ if event $B_{t, i}$ occurs and $I_{B_{t, i}}=0$ otherwise. Since the delay of a busy VODL at time $t$ is set as $i$ if and only if there is at least one arrival in $[t-i+1, t]$ that requests delay $i$ and is admitted into the flexible delay line, we have from the independent uniform delay distribution in property (ii) and $\operatorname{Pr}($ an arrival requesting delay $i$ is admitted $)=$ $p_{i}=(d /(d+1))^{d-i}$ that

$$
\begin{aligned}
\operatorname{Pr}\left(B_{t, i}\right)= & \operatorname{Pr}(\text { at least one arrival in }[t-i+1, t] \\
& \text { that requests delay } i \text { and is admitted }) \\
\approx & \operatorname{Pr}(\text { at least one arrival in }[t-i+1, t] \\
& \quad \text { that requests delay } i) \\
& \times \operatorname{Pr}(\text { an arrival requesting delay } i \text { is admitted }) \\
= & \left(1-(d /(d+1))^{i}\right) \cdot(d /(d+1))^{d-i} .
\end{aligned}
$$

We note that the event that there is at least one arrival in $[t-i+1, t]$ that requests delay $i$ and the event that an arrival requesting delay $i$ is admitted into the flexible delay line are not independent. Thus, (11) is an approximation. It then follows from $N(t)=\sum_{i=1}^{d} I_{B_{t, i}}$ and (11) that

$$
\begin{aligned}
E[N(t)] & =E\left[\sum_{i=1}^{d} I_{B_{t, i}}\right]=\sum_{i=1}^{d} \operatorname{Pr}\left(B_{t, i}\right) \\
& \approx d+1-(2 d+1)(d /(d+1))^{d} .
\end{aligned}
$$

By using $\left(1+\frac{z}{n}\right)^{n} \approx e^{z}$ for large $n$, we can see that $E[N(t)] \approx$ $\left(1-2 e^{-1}\right) d+1-e^{-1}=0.26424 d+0.63212$ for large $d$, which is much smaller than the worst-case bound $\lfloor(2 d+1) / 3\rfloor$.

In Figure 3, we show our simulation result on the average number of busy VODLs for the uniform traffic model. The simulation time is $10^{8}$ time slots. In Figure 3, we also show the worst-case bound in Theorem 1 and the approximation in (12). Note that the approximation in (12) is quite close to the simulation result.

Furthermore, we show in Figure 4 the complementary distribution of the number, say $N$, of busy VODLs. It can be seen from Figure 4 that if we can tolerate a loss probability of, say $10^{-3}$, then we only need at most 1.7 times of the average number of busy VODLs, which is approximately $0.45 d$ for large $d$ and is much smaller than the worst-case bound $\lfloor(2 d+1) / 3\rfloor$.

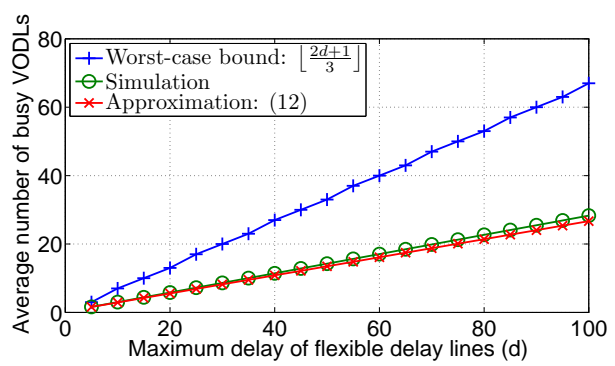

Fig. 3. Average number of busy VODLs.

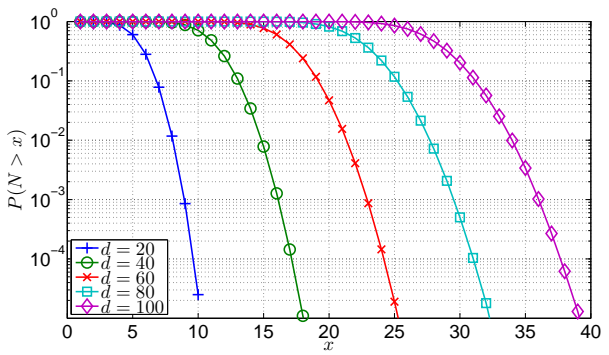

Fig. 4. Complementary distribution of the number, say $N$, of busy VODLs.

\section{CONCLUSION}

In this letter, we have proposed a construction of an optical flexible delay line by parallel variable optical delay lines (VODLs). We have obtained a worst-case bound on the number of VODLs in our proposed architecture and have shown through simulations that the number of busy VODLs could be much smaller than the worst-case bound.

\section{REFERENCES}

[1] C.-S. Chang and D.-S. Lee, Principles, Architectures and Mathematical Theory of High Performance Packet Switches, Taiwan, R.O.C.: National Tsing Hua University Press, 2008.

[2] Y.-T. Chen, J. Cheng, and D.-S. Lee, "Constructions of linear compressors, nonovertaking delay lines, and flexible delay lines for optical packet switching," IEEE/ACM Transactions on Networking, vol. 17, pp. 2014 2027, December 2009.

[3] L. Christen, O. F. Yilmaz, S. Nuccio, X. Wu, I. Fazal, A. E. Willner, C. Langrock, and M. M. Fejer, "Tunable 105 ns optical delay for 80 $\mathrm{Gb} / \mathrm{s}$ RZ-DQPSK, $40 \mathrm{~Gb} / \mathrm{s} \mathrm{RZ} / \mathrm{DPSK}$, and $40 \mathrm{~Gb} / \mathrm{s}$ RZ-OOK signals using wavelength conversion and chromatic dispersion," Optical Letters, vol. 34, pp. 542-544, February 2009. 